Research Article

\title{
Research on the Stress Distribution Law of Fully Anchored Bolt and Analysis of Influencing Factors under the Condition of Surrounding Rock Deformation
}

\author{
Chengxing Zhao $\mathbb{i D}^{1,2,3}$ Yingming Li $\mathbb{D}^{1},{ }^{1,2,3}$ Gang Liu, ${ }^{1,2,3,4}$ Dengguo Chen, ${ }^{1,2,3}$ \\ and Xiangrui Meng ${ }^{1,2,3}$ \\ ${ }^{1}$ State Key Laboratory of Mining-Induced Response and Disaster Prevention and Control in Deep Coal Mines, \\ Anhui University of Science and Technology, Huainan, Anhui 232001, China \\ ${ }^{2}$ School of Mining and Safety Engineering, Anhui University of Science and Technology, Huainan 232001, China \\ ${ }^{3}$ Key Laboratory of Safe and Effective Coal Mining of the Ministry of Education, Anhui University of Science and Technology, \\ Huainan 232001, China \\ ${ }^{4}$ Heilongjiang Ground Pressure and Gas Control in Deep Mining Key Laboratory, \\ Heilongjiang University of Science and Technology, Harbin 150022, China
}

Correspondence should be addressed to Yingming Li; libo_1296@126.com

Received 5 July 2020; Revised 23 September 2020; Accepted 30 September 2020; Published 12 October 2020

Academic Editor: Fengqiang Gong

Copyright (c) 2020 Chengxing Zhao et al. This is an open access article distributed under the Creative Commons Attribution License, which permits unrestricted use, distribution, and reproduction in any medium, provided the original work is properly cited.

\begin{abstract}
As the mining depth increases, the deformation of the roadway becomes more difficult to control. As a main supporting structure for maintaining the stability of roadway, the fully anchored bolt is widely used to reinforce deep mine. At the same time, the analysis of the stress distribution law of fully anchored bolt is the basic work to optimize anchor design. Therefore, this paper establishes a fully anchored bolt-surrounding rock interaction model based on the law of surrounding rock deformation and derives the analytical expressions for the axial force and shear stress of the fully anchored bolt during normal support and critical failure. At the same time, the effects of surrounding rock properties, support resistance, and bolt length on the stress distribution of fully anchored bolt are analyzed. The results show that the stress distribution of fully anchored bolt is consistent with the "neutral point" theory and the most important is the fact that the conditions of surrounding rock, the supporting resistance, and the length of bolt affect the actual stress distribution of the fully anchored bolt. It provides a certain theoretical basis for the design and development of anchoring and supporting technology.
\end{abstract}

\section{Introduction}

As one of the important branches of the field of geotechnical engineering, anchoring support technology is widely used in defense engineering, mining engineering, and water conservancy engineering due to its effective support effect, low cost, and convenient construction [1-9]. Especially in mining engineering, due to the increasing mining depth of coal mines in China, the maximum mining depth has reached $1500 \mathrm{~m}[10,11]$, resulting in increasingly complex surrounding rock conditions, and the carrying capacity of anchors needs to be continuously strengthened. At the same time, due to the impact of in situ stress, the surrounding rock of the roadway will interact with the anchor bolt, and the anchor bolt will restrain the surrounding rock around the roadway in the form of active support. However, the fully anchored bolt is subjected to complex forces during the support process, resulting in the development of anchoring technology theory and relatively slow anchoring design [12-14]; anchoring support technology lacks theoretical basis. The stress characteristics of the fully anchored bolt and the stress distribution law at the 
anchoring interface are important contents in anchoring support technology.

Many scholars have done a lot of research work on the stress characteristics and stress distribution laws of fully anchored bolt through numerical simulation, pull-out tests, and actual field measurements [15-21]. Freeman made a groundbreaking research work on the study of the stress distribution laws of fully anchored bolt. Pull-out tests and field measurements were used to study the stress distribution of fully anchored bolt, and the neutral point theory and the concepts of anchoring section and drawing section were proposed, where drawing section refers to the distance from the surrounding rock surface to the neutral point along the length of the bolt. At the neutral point, the shear stress on the bolt body is $0 \mathrm{MPa}$, the axial force reaches the maximum value, the shear stress direction of the pull-out section of the bolt points to the orifice, and the shear stress direction of the anchoring section is opposite to it [22]. These theoretical concepts clearly outlined the mechanical properties of the fully anchored bolt in deformed surrounding rock of roadway. In order to analyze the stress of the fully anchored bolt during the supporting process, Chen et al. mainly analyzed the support reliability of fully anchored bolt to surrounding rock with different properties. The results show that the main source of anchoring force is the bonding force between the anchor bolt and the surrounding rock of roadway. At the same time, increasing the anchoring strength will increase the stability of the surrounding rock within the anchoring range [23]. Hyett et al. obtained the shear and axial force distribution of the anchor bolt in the fractured rock under the condition of the linear relationship between the relative shear displacement of the surrounding rock and the anchor bolt [24]. Yuan et al. used FLAC ${ }^{3 \mathrm{D}}$ to establish a numerical model of nonlayered homogeneous roof and layered weak roof roadway, analyzed the supporting effect of fully anchored bolt on these 2 roadways with different conditions, and studied the stress distribution and displacement distribution in the anchorage area [25]. Cai et al. proposed a theoretical analysis model for the stress distribution of fully anchored bolt based on the existing theoretical research, described the interaction mechanism between the bolt and the surrounding rock, and discussed the influence of the bolt length on its supporting effect [26]. You obtained the elastic solution of the shear force distribution along the body of the fully anchored bolt based on the displacement analysis of the Mindlin problem and also analyzed the stress characteristics and influencing factors of the fully anchored bolt [27]. Li and Stillborg believe that there are two types of shear stress in different directions and properties on the anchoring interface of the fully anchored bolt: one is the deformation shear stress caused by the deformation of the surrounding rock, and the other is the pull-out shear stress caused by the deformation shear stress [28].

On the basis of the aforementioned theoretical research, many scholars have also tested and studied the mechanical properties of fully anchored bolt through pull-out experiments $[29,30]$. Chen et al. designed a support plate that can detect whether the fully anchored bolt is debonded. At the same time, the pull-out experiment was performed on the full-length anchor bolt to analyze the load transfer behavior of it [31]. Soparat and Nanakorn used the element-free Galerkin method (EFG) to conduct numerical simulation analysis on the bolt pullout experiment and proposed a bolt pull-out analysis that can be carried out without redividing or preassuming the crack path; it is basically consistent with the results obtained in the past actual pull-out experiments [32]. However, the model based on the experience and data obtained from the pull-out test and field measurement does not essentially explain the actual action mechanism of the fully anchored bolt.

In summary, a lot of research results have been obtained on the stress characteristics and stress distribution law of the fully anchored bolt, but most of the above research results have simplified the anchoring effect of fully anchored bolt in the analysis process and less consideration is given to the effect of actual surrounding rock deformation on the anchoring effect of fully anchored bolt; the conclusions obtained have certain limitations. Therefore, this paper is based on the relationship between the actual surrounding rock deformation and the support resistance and the balance of the stress on the bolt, and the analytical expressions of the axial force and shear stress of the fully anchored bolt during the normal support process and critical failure are obtained; also, the distribution law of axial force and shear stress of the fully anchored bolt are obtained. At the same time, the effects of surrounding rock conditions, bolt length, and support resistance on the stress distribution of the fully anchored bolt are also discussed; a certain theoretical basis for anchoring technology and design is provided. In order to facilitate subsequent analysis, this paper first makes the following assumptions [33]:

(1) The length of the roadway is unlimited, the cross section of roadway is circular, and the deformation is a plane deformation problem.

(2) The surrounding rock is isotropic and belongs to a homogeneous and continuous body. The surrounding rock not affected by excavation is in a state of hydrostatic pressure.

(3) The anchor bolt is a linear elastic body and is arranged along the radial direction of the roadway.

\section{Analysis of Surrounding Rock Deformation Law and Its Relationship with Support Resistance}

Various supporting structures are installed in the roadway to maintain the long-term stability of the roadway; these supporting structures restrain the deformation of the roadway by providing a certain amount of supporting resistance. Therefore, in order to study the control effect of support resistance on the surrounding rock deformation of the roadway, Lu and Jiang proposed that there is a huge difference in the effectiveness of the support resistance to control the deformation of the surrounding rock in the roadway of different lithology by studying the measured data 
of Huainan, Pingdingshan, and other mining areas in China $[34,35]$. The relationship between the deformation of surrounding rock surface and the support resistance is as follows:

$$
U_{0}=a p^{-b}
$$

where $U_{0}$ is the deformation of surrounding rock on roadway surface $(\mathrm{mm}), p$ is the support resistance $(\mathrm{MPa})$, and parameters $a$ and $b$ depend on the types of the surrounding rock, as shown in Table 1.

However, when the fully anchored bolts support the roadway, the bolt body must be inserted into the surrounding rock to achieve the purpose of support; in order to analyze the stress characteristics of the fully anchored bolt, we must understand the relationship between the deep deformation of rock mass and the deformation of surrounding rock on roadway surface during the support of the fully anchored bolt. Scholars have achieved many important research results on this issue. Among them, Lu et al. proposed the important indicator of deep surface ratio, which represents the ratio of the deep displacement of rock mass to the deformation of surrounding rock surface [36] (Figure 1), as follows:

$$
\eta_{r}=\frac{U_{r}}{U_{0}}
$$

where $U_{r}$ is the displacement of deep rock mass $(\mathrm{mm})$ and $\eta_{r}$ is the deep surface ratio; it takes the maximum value on the surrounding rock surface of the roadway and attenuates according to the negative exponential curve from the roadway surface to the depth of the surrounding rock. The curve equation is as follows:

$$
\eta_{r}=\mathrm{e}^{-c\left(r-a_{0}\right)}
$$

where $a_{0}$ is the radius of roadway (m), $r$ is the distance from center of roadway to a certain point in the surrounding rock $(\mathrm{mm})$, and $c$ is the stability coefficient of the rock mass.

By substituting equations (1) and (3) into equation (2), the deep displacement $U_{r}$ of rock mass is expressed as

$$
U_{r}=a p^{-b} \cdot e^{-c\left(r-a_{0}\right)} .
$$

Now suppose that the difference $F_{(r)}$ between the displacement $U_{r}$ of the rock mass without the support of the fully anchored bolt and the actual displacement $U_{a}(r)$ of the rock mass with the support of the fully anchored bolt is as follows:

$$
F_{(r)}=U_{r}-U_{a}(r)
$$

Assume that there is no relative displacement between the bolt body and the surrounding rock as follows:

$$
U_{b}=U_{a}(r),
$$

where $U_{b}$ is the displacement of the fully anchored bolts (mm).

By substituting equation (6) into equation (5), the difference $F_{(r)}$ between the displacement of the rock mass without the support of the fully anchored bolt and the actual displacement of the rock mass with the support of the fully anchored bolt is expressed as

$$
F_{(r)}=U_{r}-U_{b}
$$

and $F_{(r)}$ is expressed as a power series as follows:

$$
F_{(r)}=n_{1}+n_{2} r+n_{3} r^{2}+n_{4} r^{3}
$$

Combining equations (4), (7), and (8), the displacement $U_{\mathrm{b}}$ of the fully anchored bolt can be expressed as follows:

$$
U_{b}=a p^{-b} \cdot e^{-c\left(r-a_{0}\right)}-n_{1}-n_{2} r-n_{3} r^{2}-n_{4} r^{3} .
$$

\section{Analysis and Calculation of Stress Distribution Law of Fully Anchored Bolt}

3.1. Establishment of Mechanical Model of Fully Anchored Bolt. In order to analyze the stress of the fully anchored bolt during support process, the microunit of the fully anchored bolt is taken as shown in Figure 2. According to the force balance relationship of the fully anchored bolt, the relationship between the axial force and the shear force on the surface of the bolt body can be shown as follows:

$$
\mathrm{d} N=T \mathrm{~d} r,
$$

where $N$ is the axial force on the fully anchored bolt $(\mathrm{kN})$ and $T$ is the shear force on the surface of the microunit of the fully anchored bolt $(\mathrm{kN})$.

According to Hooke's law, the axial force $N$ on the fully anchored bolt can be obtained as follows:

$$
N=A_{a} E_{a} \frac{\mathrm{d} U_{b}}{\mathrm{~d} r}
$$

where $A_{\mathrm{a}}$ is the converted cross-sectional area of the anchor bolt and $E_{\mathrm{a}}$ is the elastic modulus of the fully anchored bolt, which can be expressed as follows:

$$
A_{a}=A_{s}+A_{c} \frac{E_{a}}{E_{c}}
$$

where $A_{\mathrm{s}}$ is the cross-sectional area of the fully anchored bolt, $A_{\mathrm{c}}$ is the cross-sectional area of the anchoring agent, and $E_{\mathrm{c}}$ is the elastic modulus of the anchoring agent; combining equations (11) and (12), the axial force $N$ on the fully anchored bolt can be shown as follows:

$$
N=E_{a} \cdot\left(A_{s}+A_{c} \frac{E_{a}}{E_{c}}\right) \frac{\mathrm{d} U_{b}}{\mathrm{~d} r} .
$$

By substituting equation (13) into equation (10), the shear force $T$ on the surface of the microunit of the fully anchored bolt can be shown as follows:

$$
T=E_{a} \cdot\left(A_{s}+A_{c} \frac{E_{a}}{E_{c}}\right) \frac{\mathrm{d}^{2} U_{b}}{\mathrm{~d} r^{2}} .
$$

The relationship between the shear stress and shear force on the fully anchored bolt can be expressed as follows: 
TABLE 1: The coefficients $a$ and $b$ of surrounding rock.

\begin{tabular}{lcc}
\hline Types of surrounding rock & $a$ & $b$ \\
\hline Soft rock & 9.20 & 1.190 \\
Medium hard rock & 2.14 & 1.160 \\
Hard rock & 0.53 & 1.020 \\
\hline
\end{tabular}

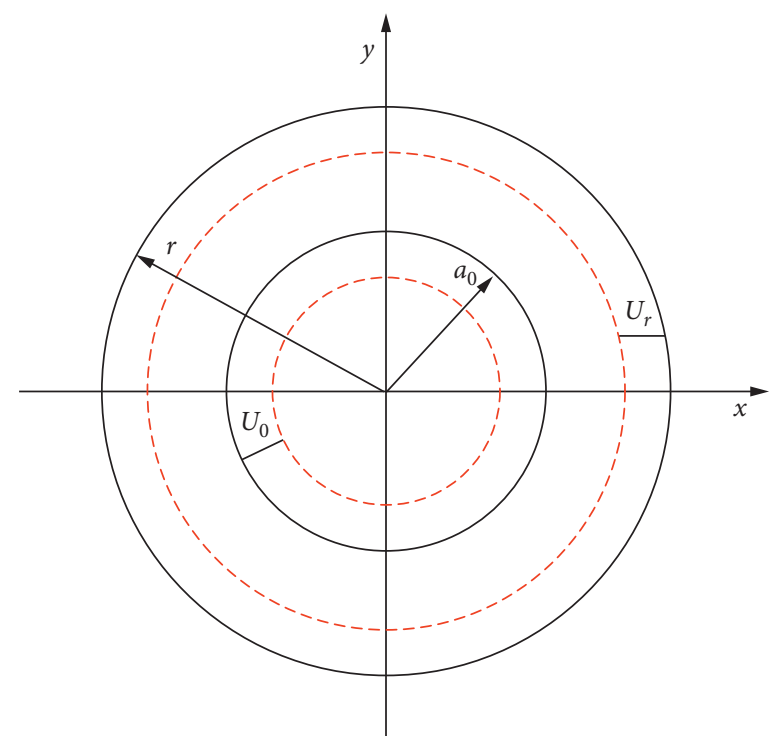

FIGURE 1: The relationship between the deformation of surrounding rock on roadway surface and the displacement of deep rock mass.

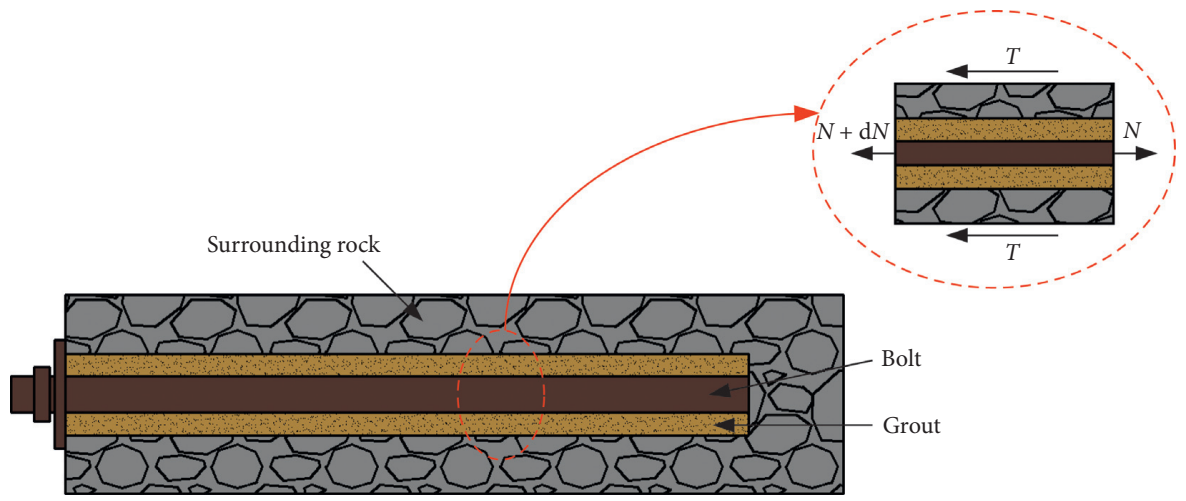

Figure 2: The microunit of the fully anchored bolt.

$$
\tau=\frac{T}{\pi d}
$$

where $\tau$ is the shear stress on the fully anchored bolt ( $\mathrm{MPa}$ ) and $d$ is the fully anchored bolt cross-sectional diameter (m). The balance between the shearing force $T$ of the fully anchored bolt and the reaction force $Q$ of the pallet can be expressed as follows:

$$
Q+\int_{a_{0}}^{a_{0}+L} T \mathrm{~d} r=0,
$$

where $L$ is the length of the fully anchored bolt (m). At the same time, combining equations (9), (13), (14), and (15), the axial force $N$ and the shear stress $\tau$ on the fully anchored bolt can be expressed, respectively, as follows:

$$
\begin{aligned}
N= & E_{a} \cdot\left(A_{s}+A_{c} \frac{E_{a}}{E_{c}}\right) \times\left(-c \cdot a p^{-b} e^{-c\left(r-a_{0}\right)}-n_{2}\right. \\
& \left.-2 n_{3} r-3 n_{4} r^{2}\right), \\
\tau= & \frac{E_{a} \cdot\left(A_{s}+A_{c}\left(E_{a} / E_{c}\right)\right) \times\left(c^{2} \cdot a p^{-b} e^{-c\left(r-a_{0}\right)}-2 n_{3}-6 n_{4} r\right)}{\pi d} .
\end{aligned}
$$

In the normal support process of the fully anchored bolt, due to the different deformation of the surrounding rock at different depths, the shear direction of each part of the fully anchored bolt is not exactly the same. Some shear forces are in the same direction as the reaction force of the tray and 
point to the direction of the orifice and the sum balanced with the shear force directed to the end of the anchoring section; there is a neutral point on the bolt, that is, the fully anchored bolt with neutral point, as shown in Figure 3(a). At the neutral point, the shear stress is $0 \mathrm{MPa}$ and the axial force reaches the maximum value.

As the deformation of surrounding rock increases continuously, the strength of support cannot effectively suppress the deformation of the roadway, and the bolt and the surrounding rock will slide relative to each other. When the support fails critically, the direction of the shear force on the bolt will point to its end and be balanced with the reaction force $Q$ of the pallet; the shear stress at the orifice and the bolt end is $0 \mathrm{MPa}$, that is, the fully anchored bolt without neutral point, as shown in Figure 3(b).
Therefore, firstly analyzing the stress condition of the anchored bolt in Figure 3(a), the boundary conditions can be expressed as follows:

$$
\begin{cases}r=a_{0}+r_{n}, & \tau=0, \\ r=a_{0}+L, & \tau=0, \\ r=a_{0}+L, & N=0,\end{cases}
$$

where $r_{n}$ is the radius of the neutral point, which represents the distance from the orifice to the neutral point.

By substituting equation (19) into equations (17) and (18), the parameters $n_{2}, n_{3}$, and $n_{4}$ can be expressed, respectively, as follows:

$$
\left\{\begin{array}{l}
n_{2}=-c \cdot a p^{-b} e^{-c L}-\left(a_{0}+L\right) \cdot\left(c^{2} \cdot a p^{-b} e^{-c r_{n}}-\frac{c^{2} \cdot a p^{-b} \cdot\left(a_{0}+r_{n}\right)\left(e^{-c r_{n}}-e^{-c L}\right)}{r_{n}-L}\right) \\
-\frac{c^{2} \cdot a p^{-b}\left(e^{-c r_{n}}-e^{-c L}\right) \cdot\left(a_{0}+L\right)^{2}}{2\left(r_{n}-L\right)} \\
n_{3}=\frac{c^{2} \cdot a p^{-b} e^{-c L}}{2}-\frac{c^{2} \cdot a p^{-b}\left(e^{-c r_{n}}-e^{-c L}\right) \cdot\left(a_{0}+r_{n}\right)}{2\left(r_{n}-L\right)} \\
n_{4}=\frac{c^{2} \cdot a p^{-b}\left(e^{-c r_{n}}-e^{-c L}\right)}{6\left(r_{n}-L\right)},
\end{array}\right.
$$

The analytical formula for the axial force and shear stress of the fully anchored bolt can be obtained, as well as the analytical formula for shear stress calculation; and equation
(15) can be substituted into equation (16) to obtain the relationship between the pallet reaction force $Q$ and the neutral point radius $r_{\mathrm{n}}$. It can be expressed as follows:

$$
Q=-E_{a}\left(A_{s}+A_{c} \frac{E_{a}}{E_{c}}\right) \times\left[\begin{array}{c}
a \cdot c p^{-b}\left(1-e^{-c L}-c L e^{-c r_{n}}\right)+\frac{a_{0} c^{2} \cdot a p^{-b}\left(a_{0}+r_{n}\right)\left(e^{-c r_{n}}-e^{-c L}\right)}{r_{n}-L} \\
-\frac{a p^{-b} \cdot c^{2}\left(e^{-c r_{n}}-e^{-c L}\right)\left(L^{2}+2 a_{0} L\right)}{2\left(r_{n}-L\right)}
\end{array}\right] .
$$

For the stress condition of the fully anchored bolt in Figure 3(b), the boundary conditions can be expressed as follows:

$$
\begin{cases}r=a_{0}, & \tau=0, \\ r=a_{0}+L, & \tau=0, \\ r=a_{0}+L, & N=0\end{cases}
$$




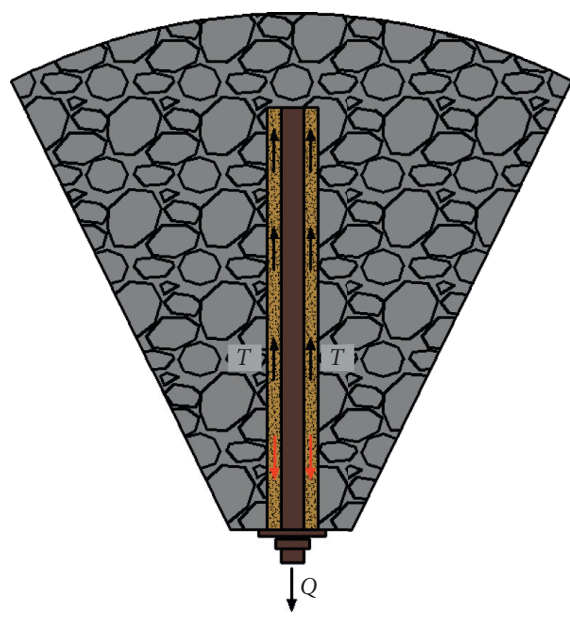

(a)

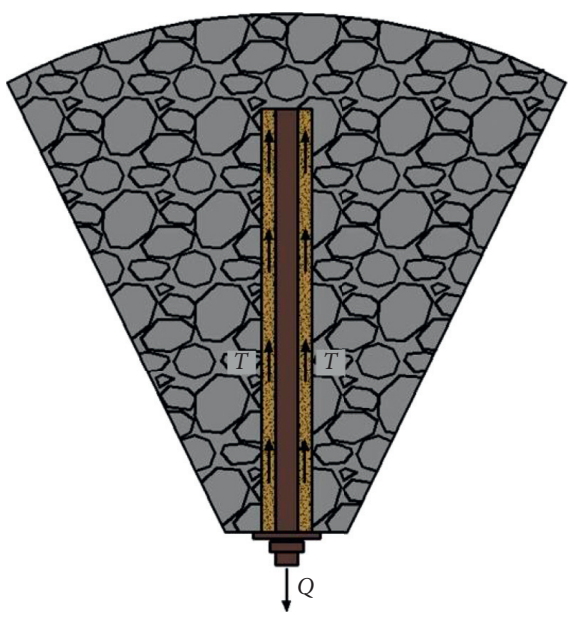

(b)

FIGURE 3: Stress condition of fully anchored bolt. (a) The fully anchored bolt with neutral point. (b) The fully anchored bolt without neutral point.

Similarly, by substituting equation (22) into equations (17) and (18), the parameters $n_{2}, n_{3}$, and $n_{4}$ can be obtained, which can be expressed as follows:
TABLE 2: The stability coefficient $c$ of surrounding rock.

\begin{tabular}{lccc}
\hline $\begin{array}{l}\text { Types of surrounding } \\
\text { rock }\end{array}$ & $\begin{array}{c}\text { Soft } \\
\text { rock }\end{array}$ & $\begin{array}{c}\text { Medium hard } \\
\text { rock }\end{array}$ & $\begin{array}{c}\text { Hard } \\
\text { rock }\end{array}$ \\
\hline$C$ & 0.65 & 0.95 & 1.35 \\
\hline
\end{tabular}

$$
\left\{\begin{array}{l}
n_{2}=-c \cdot a p^{-b} e^{-c L}-\left(a_{0}+L\right) \cdot\left(c^{2} \cdot a p^{-b}-\frac{a_{0} c^{2} \cdot a p^{-b}\left(e^{-c L}-1\right)}{L}\right) \\
-\left(a_{0}+L\right)^{2} \frac{c^{2} \cdot a p^{-b}\left(e^{-c L}-1\right)}{2 L} \\
n_{3}=\frac{c^{2} \cdot a p^{-b}}{2}-\frac{a_{0} c^{2} \cdot a p^{-b}\left(e^{-c L}-1\right)}{2 L} \\
n_{4}=\frac{c^{2} \cdot a p^{-b}\left(e^{-c L}-1\right)}{6 L}
\end{array}\right.
$$

By substituting equation (23) into equations (17) and (18), analytical formulas for calculating the axial force and shear stress of the fully anchored bolt under the second stress condition can be obtained. From the above analysis, it can be seen that the surrounding rock conditions, anchoring parameters, and support resistance affect the stress distribution law of the fully anchored bolt. Therefore, this paper will analyze the stress distribution of the fully anchored bolt under two different working conditions of it.

3.2. Analysis of the Stress Distribution Law of the Fully Anchored Bolt during Normal Support Process. It can be seen from equations (17) and (18) that the anchoring parameters, support resistance, and surrounding rock conditions affect the stress distribution of the fully anchored bolt. In this paper, the effects of surrounding rock conditions, support resistance, and the length of bolt on the stress distribution law of the fully anchored bolt are now analyzed, respectively.

3.2.1. Influence of the Surrounding Rock Conditions on the Stress Distribution Law of the Fully Anchored Bolt during Normal Support Process. In order to analyze the stress distribution law of the fully anchored bolt under different surrounding rock conditions, we assume that the radius of the roadway is $3.0 \mathrm{~m}$, the cross-sectional area of the anchoring agent is $6.158 \times 10^{-4} \mathrm{~m}^{2}$, and its elastic modulus is $17 \mathrm{GPa}$; for analysis of the influence of the different surrounding rock conditions on the stress distribution law, the value of the support resistance is set as $0.2 \mathrm{MPa}$, the length of the bolt is $2.2 \mathrm{~m}$, and its cross-sectional area and elastic modulus are $4.155 \times 10^{-4} \mathrm{~m}^{2}$ and $200 \mathrm{GPa}$, respectively. However, the stability coefficient $c$ of surrounding rock is 


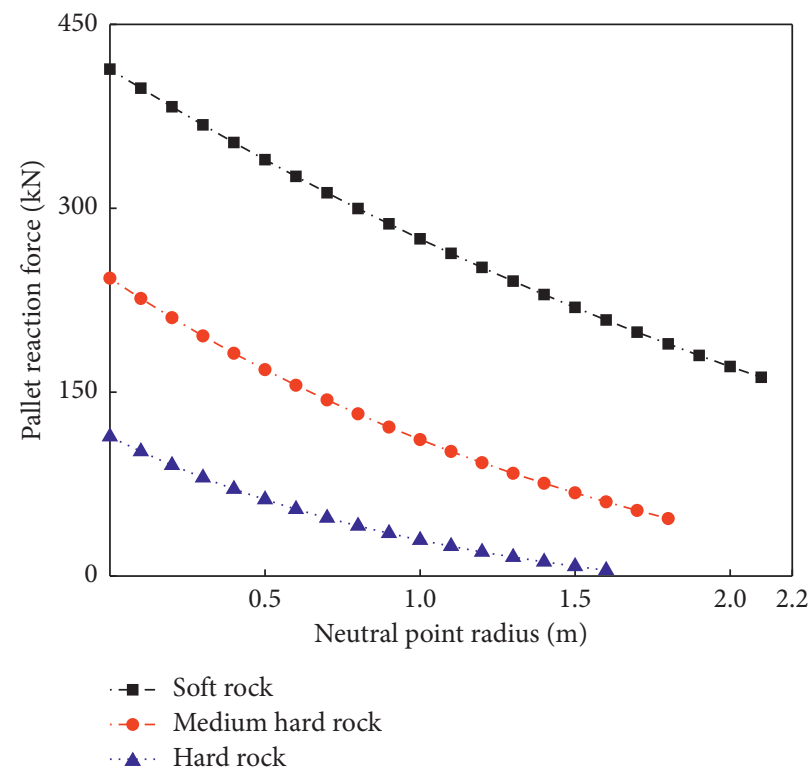

FIgURE 4: The relationship curve between the neutral point radius $r_{n}$ and the pallet reaction force $Q$ under different surrounding rock conditions.

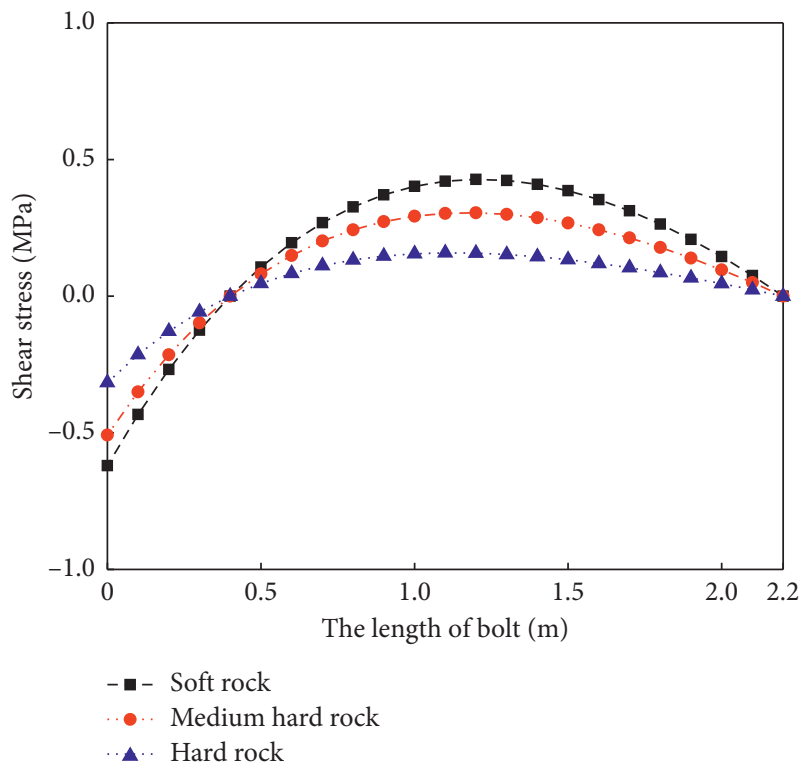

(a)

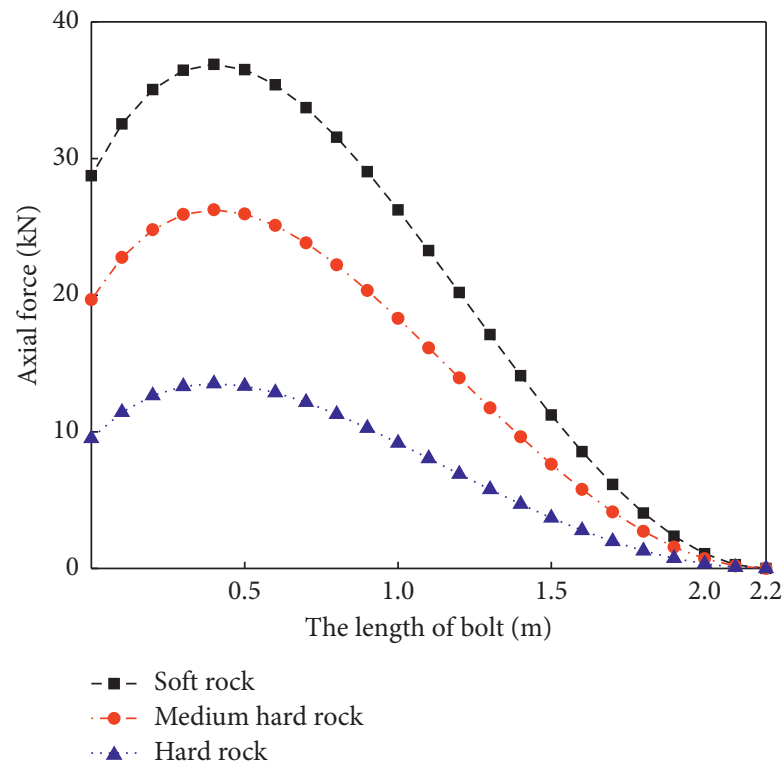

(b)

FiguRE 5: The curves of stress distribution of the fully anchored bolt under different surrounding rock conditions. (a) The curve of shear stress. (b) The curve of axial force.

not consistent under different surrounding rock conditions. Yang et al. [35] assigned this parameter, as shown in Table 2.

Therefore, we substitute the values of the above parameters into equation (21) to obtain the relationship curve between the neutral point radius $r_{n}$ on the fully anchored bolt and the pallet reaction force $Q$ under different surrounding rock conditions, as shown in Figure 4.

From Figure 4, we can understand that as the reaction force of the pallet increases, the radius of the neutral point will decrease and the neutral point will move toward the orifice under the same surrounding rock conditions. The harder the surrounding rock is, the greater the neutral point movement range will be, and the overall pallet reaction force becomes smaller. The main reason is that the softer the surrounding rock is, the serious its deformation will be, the greater the force of the fully anchored bolt will be, and the greater the reaction force of the pallet will be. At the same time, due to the different types of the surrounding rock, the actual change range of the pallet reaction force is also different, which makes it impossible for us to analyze the 


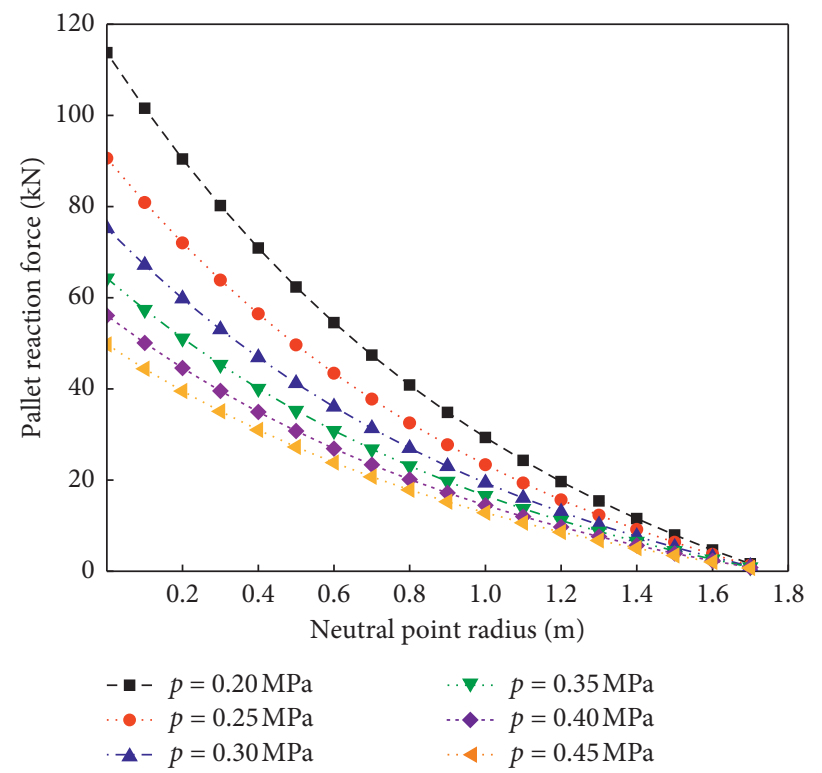

FIGURE 6: The relationship curve between the neutral point radius $r_{n}$ and the pallet reaction force $Q$ under different support resistances.

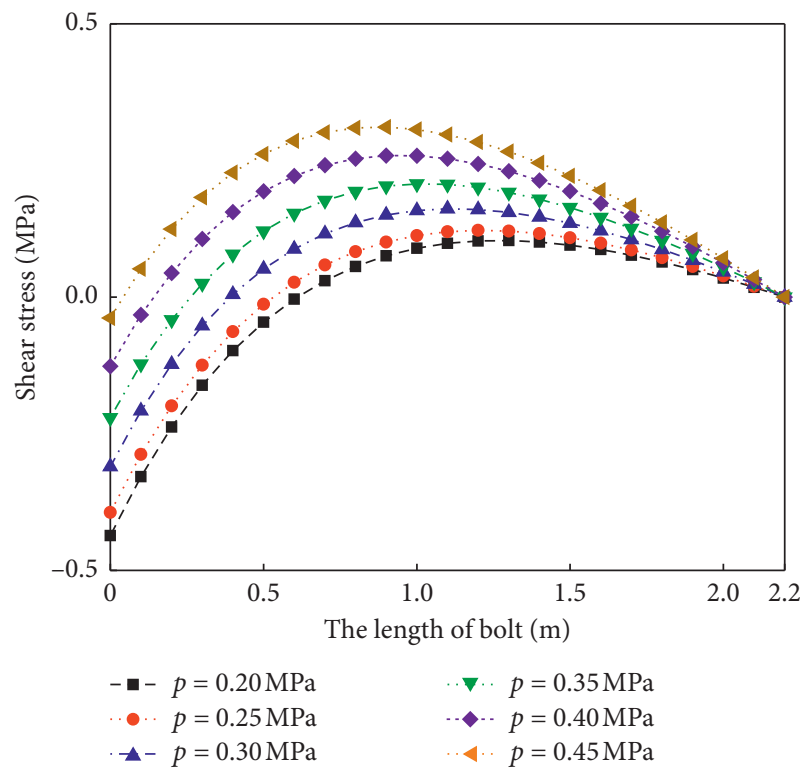

(a)

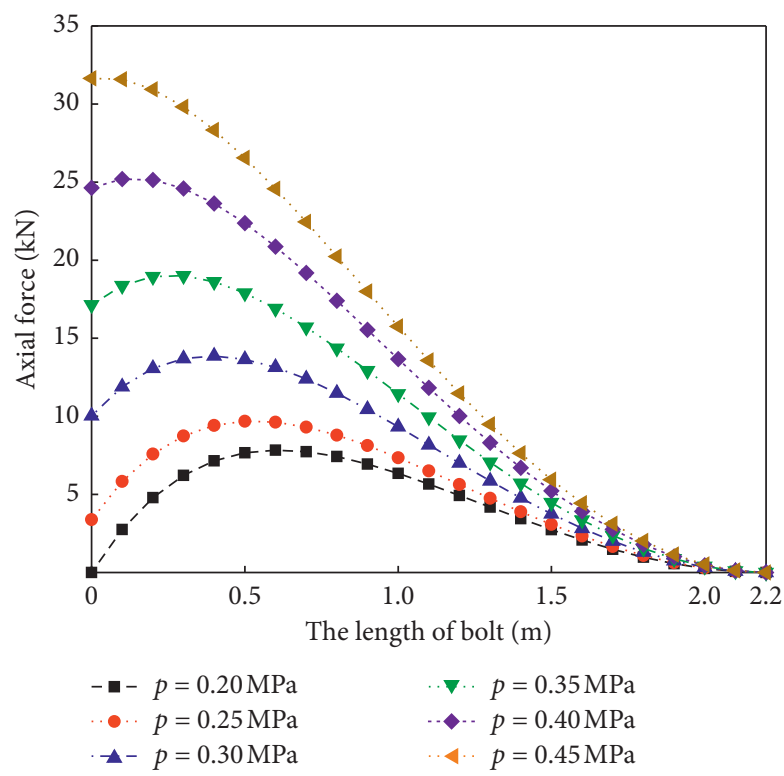

(b)

FIgURE 7: The curves of stress distribution of the fully anchored bolt under the conditions of different support resistances. (a) The curve of shear stress. (b) The curve of axial force.

influence of the lithology on the stress distribution of the fully anchored bolt.

In order to analyze the stress distribution law of the fully anchored bolt during normal support under different surrounding rock conditions, we now assume that the radius of the neutral point under different surrounding rock conditions is $0.4 \mathrm{~m}$ and then obtain the stress distribution curve of the bolt under different surrounding rock conditions, as shown in Figure 5, respectively.

From Figure 5, it can be seen that, no matter what kind of rock mass, the stress distribution law of the fully anchored bolt is consistent with the neutral point theory in normal support process. At the neutral point, the shear stress on the bolt is $0 \mathrm{MPa}$, and the axial force has the maximum value. The most important is that the lithology of the surrounding rock affects the stress distribution law of the fully anchored bolt; as the surrounding rock becomes softer, the axial force and shear stress on the anchor bolt increase. The main reason for this is that the harder the surrounding rock is, the smaller the deformation of the surrounding rock will be and the less chance that the anchored bolt will participate in suppressing the deformation of the surrounding rock. 
Therefore, when selecting anchored bolts to support the roadway, the lithology and other relevant factors of the surrounding rock should be considered.

3.2.2. Influence of the Support Resistance on the Stress Distribution Law of the Fully Anchored Bolt during Normal Support Process. In order to explore the influence of the support resistance on the stress distribution law of the fully anchored bolt during normal support, except for the surrounding rock conditions and the support resistance, other relevant parameters should be consistent with the above example to obtain the relationship curve between the pallet reaction force $Q$ and the neutral point radius $r_{n}$, as shown in Figure 6. Therefore, we assume that the condition of surrounding rock is hard rock and set the support resistance as

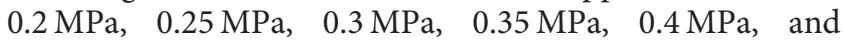
$0.45 \mathrm{MPa}$, respectively.

From Figure 6, it is clear that the movement range of the neutral point is the part with a distance of $1.7 \mathrm{~m}$ from the orifice under the condition of different support resistance. It indicates that the increase or decrease of the overall support resistance does not affect the movement range of the neutral point during the normal support process of the bolt, but as the reaction force of the pallet increases, the radius of the neutral point continues to decrease, and the neutral point gradually moves toward the orifice. When the neutral point is at the orifice position, the maximum reaction force of the pallet appears. However, with the increase of the overall support resistance, the variation range of the pallet reaction force has been continuously reduced. Therefore, we set the pallet reaction force as $48 \mathrm{kN}$ to explore the influence of the change of support resistance on the stress distribution of the fully anchored bolt, as shown in Figure 7 .

The curves of stress distribution of the fully anchored bolt under different support resistance are plotted in Figure 7. It is clear that the support resistance has a certain influence on the stress distribution of the fully anchored bolt. When the support resistance is $0.45 \mathrm{MPa}$, the maximum axial force and shear stress on the bolt are $31.6 \mathrm{kN}$ and $0.31 \mathrm{MPa}$, respectively. When the support resistance is $0.20 \mathrm{MPa}$, the maximum axial force and shear stress on the bolt are $7.8 \mathrm{kN}$ and $0.1 \mathrm{MPa}$, respectively. The results show that as the support resistance increases from $0.20 \mathrm{MPa}$ to $0.45 \mathrm{MPa}$, the axial force and shear stress on the fully anchored bolt increase as a whole, the axial force and shear stress are unevenly distributed, and the neutral point gradually moves toward the orifice. The most important is the fact that the shear stress on the fully anchored bolt is unevenly distributed along the bolt body. At the position of the orifice, the shear stress is greater, and its direction points to the orifice. At the position of the neutral point, the shear stress decreases to $0 \mathrm{MPa}$, and then as it keeps away from the neutral point, the shear stress quickly increases to the maximum value and its direction points to the end of the bolt. After reaching the peak value, the shear stress decreases at a faster rate until it reaches the end of bolt. As the overall support resistance increases, the neutral point moves forward along the anchor bolt, and the shear stress distribution becomes gradually smoother. At the same time, the shear stress decreases at the orifice location, but the peak value of the shear stress becomes larger.

From Figure 7(b), it can be seen that the axial force is larger at the orifice, but as the distance from the orifice continues to increase, the axial force gradually increases to the peak value and then decreases rapidly. The increase rate of it is obviously smaller than its decrease rate, and its maximum value appears at the neutral point. As the support resistance continues to increase, the more uneven the axial force distribution is, the more the neutral point moves toward the orifice, and the axial force on the bolt also increases significantly. The above analysis shows that, during normal support of the fully anchored bolt, support resistance is an important factor affecting its stress distribution. As we all know, the spacing between the fully anchored bolts is an important factor that affects the resistance of the support of roadway. Therefore, the anchored bolts should be laid out reasonably in conjunction with other important factors such as deformation of surrounding rock in actual support.

\subsubsection{Influence of the Length of Bolt on the Stress Distribution} Law of the Fully Anchored Bolt during Normal Support Process. For the purpose of researching the influence of the bolt length on the stress distribution law of the fully anchored bolt during normal support, except for the surrounding rock conditions and the length of bolt, other relevant parameters should be consistent with the above example to obtain the relationship curve between the pallet reaction force $Q$ and the neutral point radius $r_{n}$ under the conditions of the different length of the fully anchored bolt, as shown in Figure 8. Therefore, we assume that the condition of surrounding rock is medium hard rock and set the length of bolt as $2.0 \mathrm{~m}, 2.05 \mathrm{~m}, 2.1 \mathrm{~m}, 2.15 \mathrm{~m}, 2.2 \mathrm{~m}$, and $2.25 \mathrm{~m}$, respectively.

Figure 8 illustrates that the neutral point movement range becomes larger as the length of the fully anchored bolt increases, and the value of pallet reaction force decreases overall. The value of pallet reaction force is now set as $215 \mathrm{kN}$ to obtain the stress distribution curve of the fully anchored bolt under the conditions of different length of bolt, as shown in Figure 9.

From Figure 9, it can be seen that, no matter how long the bolt is, the stress distribution of the bolt is consistent with the neutral point theory during the normal support of the fully anchored bolt. More importantly, the neutral point is always located at $1 / 4$ of the length of the bolt to the range of the orifice, the neutral point moves toward the orifice, and the stress on bolt continues to increase as the length of the bolt increases. However, the increments of axial force and shear stress decrease with the increase of the length of the bolt. Therefore, simply increasing the length of the bolt cannot significantly improve the anchoring effect of the bolt during normal support process.

3.3. Analysis of the Stress Distribution Law of the Fully Anchored Bolt during Failed Support Process. In the above analysis, it has been explained that the fully anchored bolt 


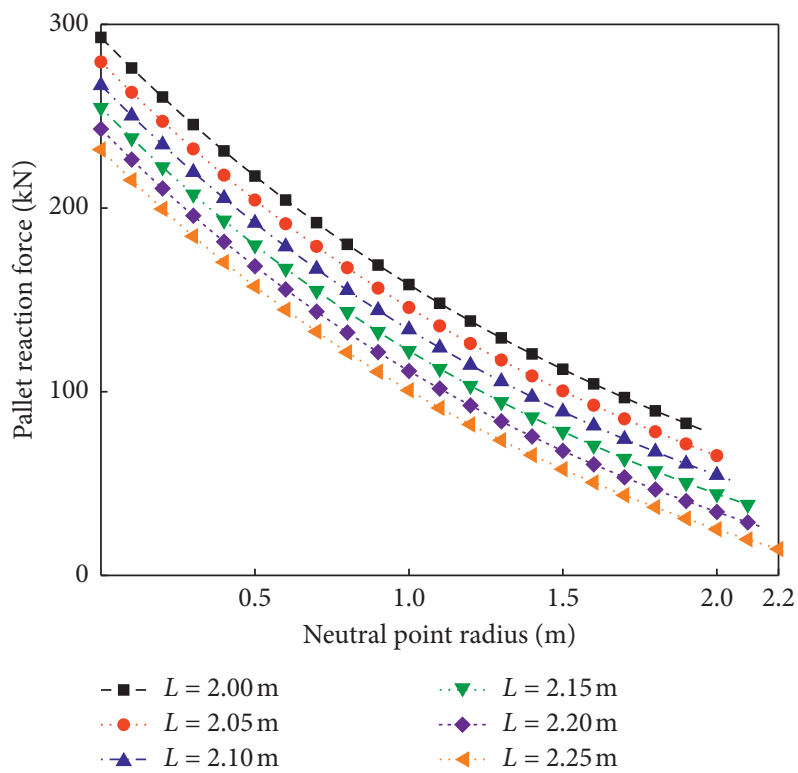

FIGURE 8: The relationship curve between the neutral point radius $r_{n}$ and the pallet reaction force $Q$ under the conditions of different length of bolt.

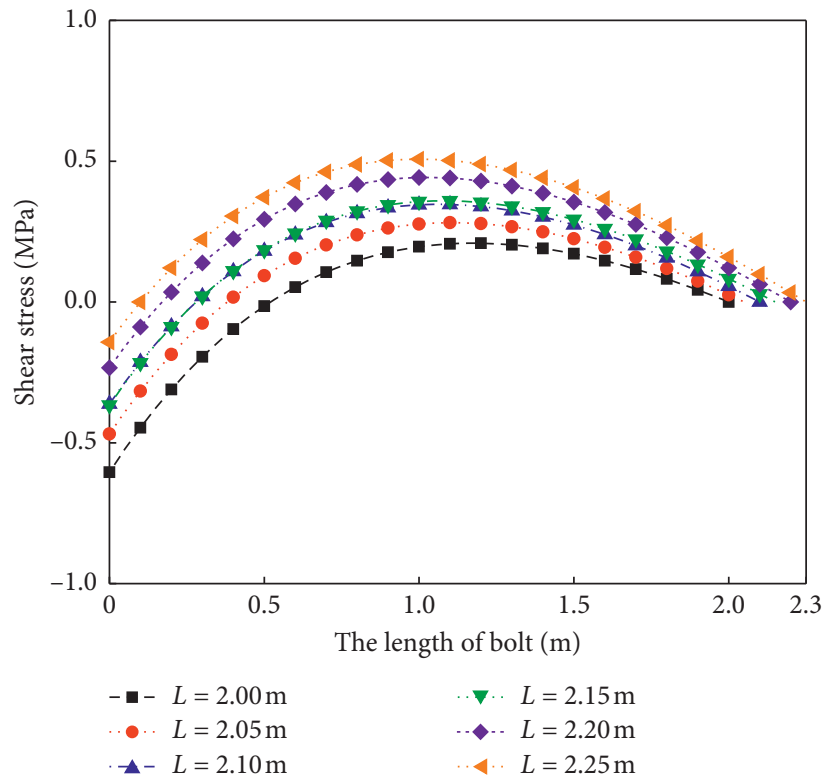

(a)

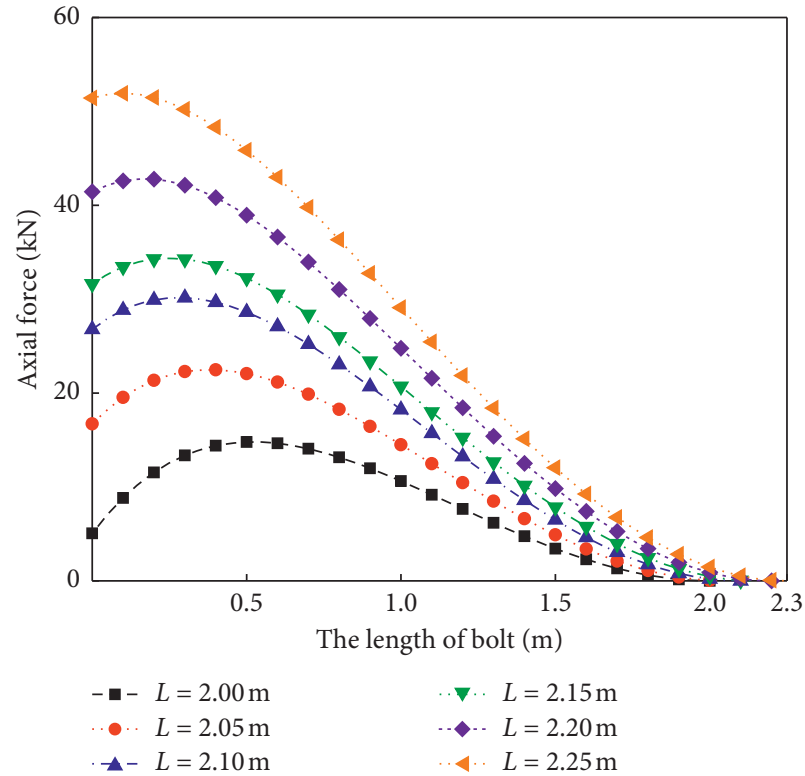

(b)

Figure 9: The curves of stress distribution of the fully anchored bolt under the conditions of different length of bolt. (a) The curve of shear stress. (b) The curve of axial force.

has two states of stress in the support process. Therefore, the stress distribution law of the fully anchored bolt during failed support process will be analyzed.

3.3.1. Influence of the Surrounding Rock Conditions on the Stress Distribution Law of the Fully Anchored Bolt during Failed Support Process. The radius of roadway and anchoring parameters are consistent with the parameters of the fully anchored bolt during normal support process under different surrounding rock conditions, so as to obtain the stress distribution curve of the fully anchored bolt when it supports critical failure under different surrounding rock conditions, as shown in Figure 10.

Figure 10 illustrates that, no matter what kind of rock mass the fully anchored bolt supports, when the bolt cannot effectively suppress the deformation of the surrounding rock of roadway, the shear stress is unevenly distributed, and the value of shear stress is $0 \mathrm{MPa}$ at the position of orifice. The shear stress rises to the peak and then quickly decays to 


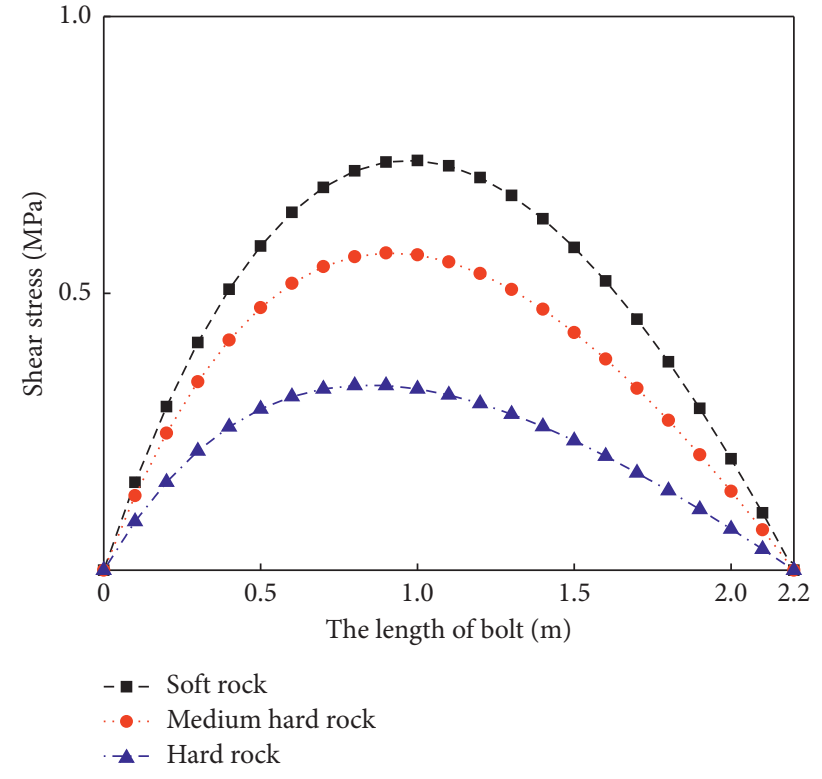

(a)

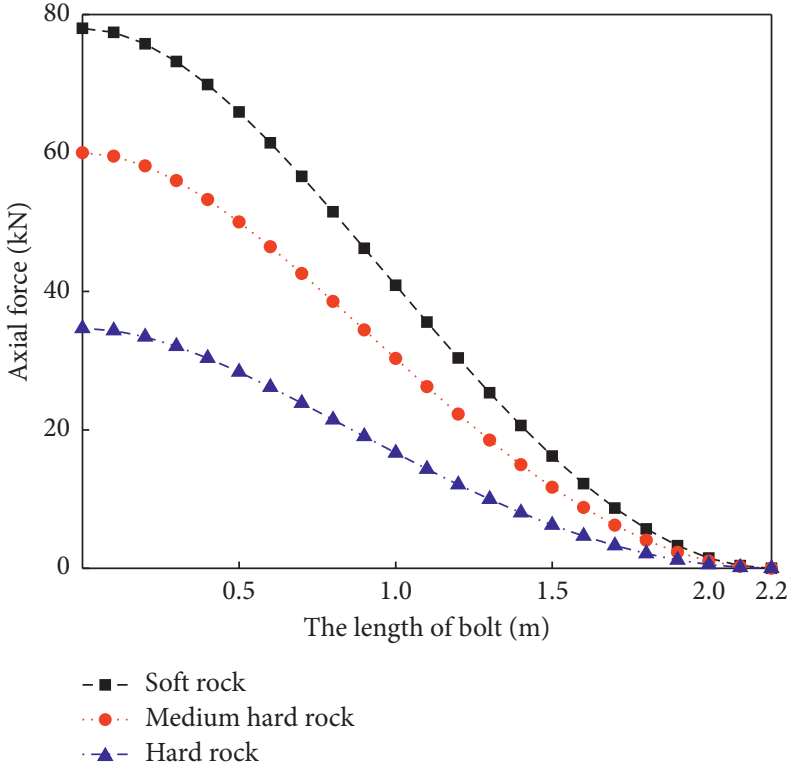

(b)

Figure 10: The curves of stress distribution of the fully anchored bolt under the conditions of different surrounding rock. (a) The curve of shear stress. (b) The curve of axial force.

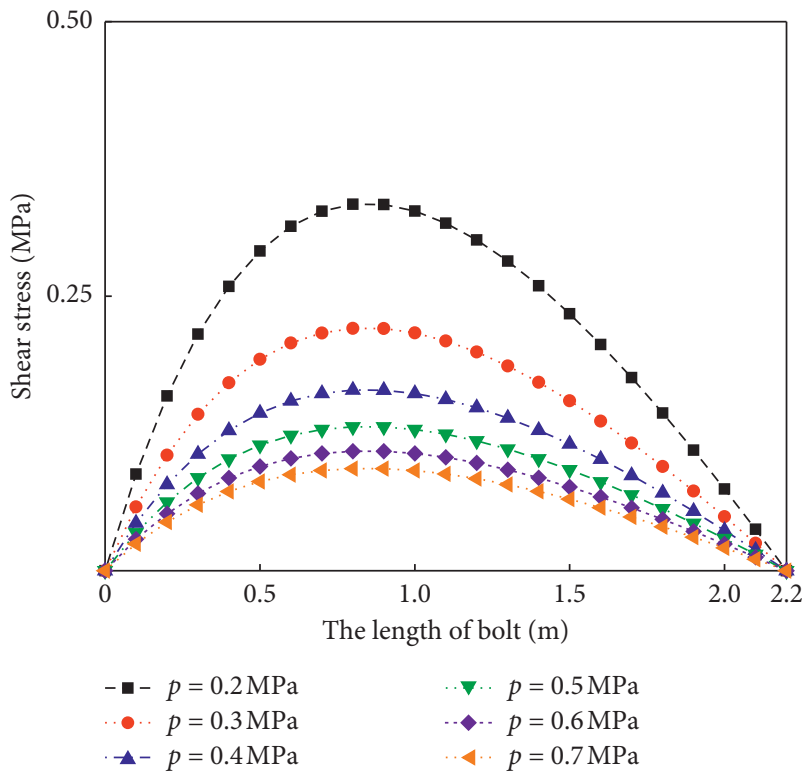

(a)

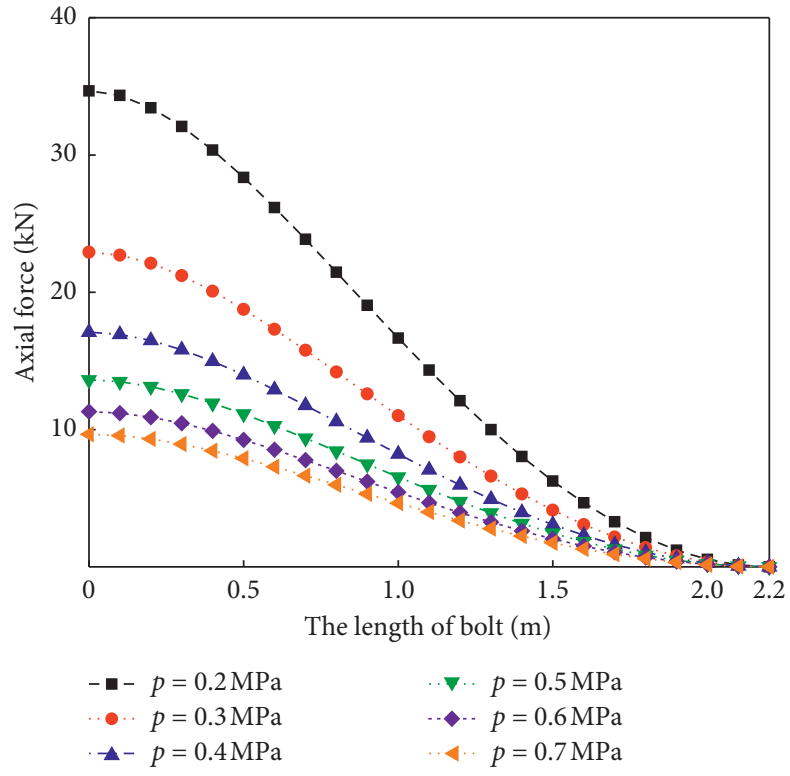

(b)

FIGURE 11: The curves of stress distribution of the fully anchored bolt under the conditions of different support resistances. (a) The curve of shear stress. (b) The curve of axial force.

$0 \mathrm{MPa}$ along the direction of the bolt length. When the fully anchored bolt supports the soft rock, the shear stress increase rate is obviously the largest, and the shear stress reaches a peak value of $0.74 \mathrm{MPa}$ at the position of $1.0 \mathrm{~m}$ from the orifice. When the fully anchored bolt supports the medium hard rock, the shear stress reaches a peak value of $0.57 \mathrm{MPa}$ at the position of $0.9 \mathrm{~m}$ from the orifice; when the fully anchored bolt supports the hard rock, the shear stress is significantly smaller, and the shear stress reaches a peak value of $0.33 \mathrm{MPa}$ at a distance of $0.8 \mathrm{~m}$ from the orifice. These data indicate that when the fully anchored bolt supports critical failure, the shear stress on the bolt increases when the rock mass becomes soft. The most obvious is the fact that the maximum shear stress on the fully anchored 


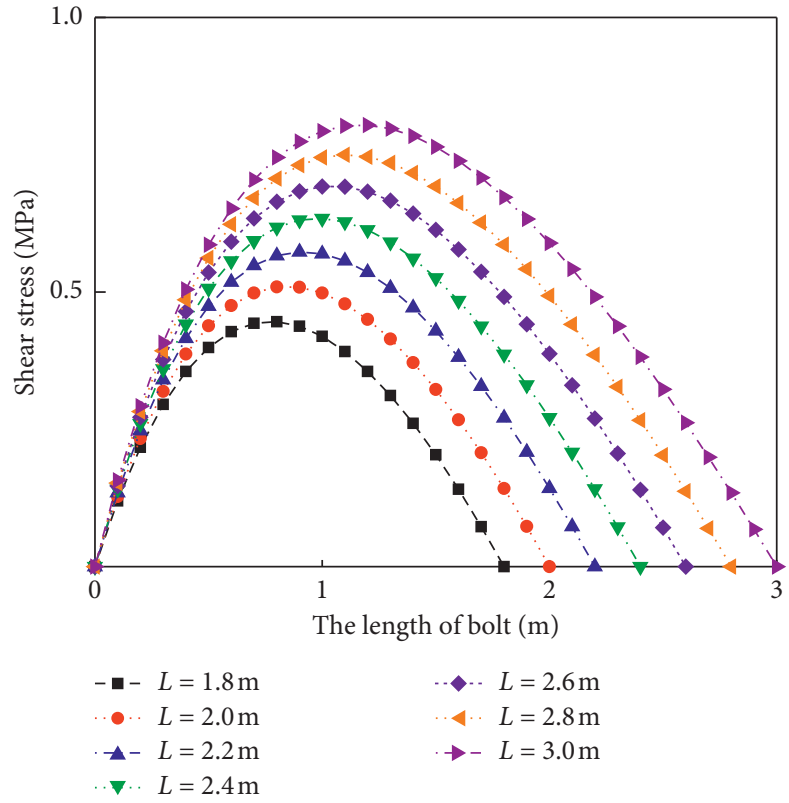

(a)

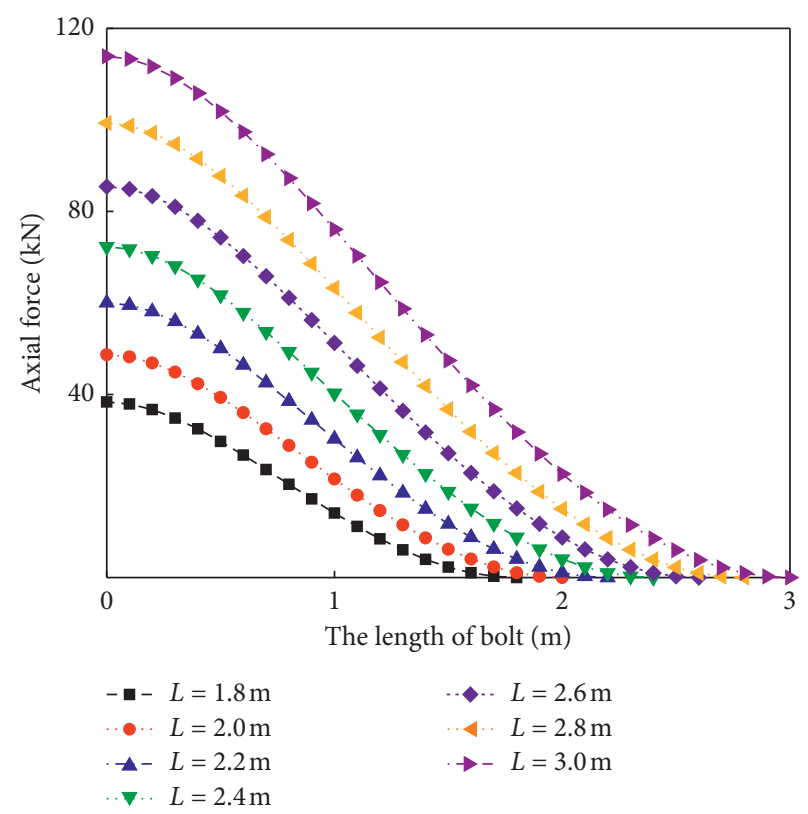

(b)

FIgURE 12: The curves of stress distribution of the fully anchored bolt under the condition of different length of bolt. (a) The curve of shear stress. (b) The curve of axial force.

bolt increases by about 1.24 times when the surrounding hard rock becomes soft rock, and the peak of shear stress also moves toward the orifice. For the distribution of the axial force of the fully anchored bolt, it can be seen from Figure 10 that when the bolt supports the soft rock, the axial force at the orifice reaches a maximum value of $78.0 \mathrm{kN}$ and then rapidly decreases in the direction of the end of the bolt until it decays to $0 \mathrm{kN}$. When the bolt supports the medium hard rock and the hard rock, respectively, the axial force distribution is similar to the axial force distribution when the bolt supports the soft rock, and the peak values of their axial forces are $60.0 \mathrm{kN}$ and $34.7 \mathrm{kN}$, respectively. The force decay rate is obviously smaller than the axial force decay rate when the bolt supports soft rock.

The above analysis shows that, in the critical failure of bolt support, serious rock deformation will cause the stress distribution of the fully anchored bolt to be more concentrated.

3.3.2. Influence of the Support Resistance on the Stress Distribution Law of the Fully Anchored Bolt during Failed Support Process. In order to explore the influence of the support resistance on the stress distribution law of the fully anchored bolt during failed support process, except for the support resistance, other relevant parameters should be consistent with the above example to obtain the stress distribution curve of the fully anchored bolt when it supports critical failure under different support resistances. Therefore, we set the support resistance as $0.2 \mathrm{MPa}, 0.3 \mathrm{MPa}, 0.4 \mathrm{MPa}, 0.5 \mathrm{MPa}$, $0.6 \mathrm{MPa}$, and $0.7 \mathrm{MPa}$, respectively, as shown in Figure 11.

From Figure 11, it can be seen that when the fully anchored bolt critically supports failure, the support resistance has a certain effect on the stress distribution of the fully anchored bolt. When the support resistance increases from $0.2 \mathrm{MPa}$ to $0.7 \mathrm{MPa}$, the shear stress and axial force on the bolt are reduced. Moreover, the shear stress is $0 \mathrm{MPa}$ at the position of the orifice and the end of the bolt, while the shear stress reaches the maximum value at the middle of the bolt, and the axial force reaches the maximum value at the position of the orifice, which is to decay until the end of the bolt decay to $0 \mathrm{kN}$ along the direction of the length of the bolt.

The most important is the fact that although the increase of the support resistance can reduce the stress of the bolt when the fully anchored bolt critically supports failure, as the support resistance increases, the total amount of stress reduction decreases continuously. This means that it is not feasible to blindly increase the support resistance to control the stability of the roadway during failed support process. Therefore, relevant supporting measures should be taken to comprehensively reinforce the surrounding rock of the roadway under the condition of considering the deformation of the surrounding rock and other factors.

\subsubsection{Influence of the Length of Bolt on the Stress Distribution} Law of Fully Anchored Bolt during Failed Support Process. The radius of roadway and some relevant parameters are consistent with the parameters of the fully anchored bolt during normal support process under the condition of different length of bolt except for the length of bolt, so as to obtain the stress distribution curve of the fully anchored bolt when it supports critical failure under the conditions of the different length of bolt, as shown in Figure 12.

It is illustrated that the axial force and shear stress are unevenly distributed on the fully anchored bolt during failed 
support process. As the length of the bolt increases, the axial force and shear stress at each position on the bolt increase, but the increments of shear stress decrease. It is shown that when the fully anchored bolt cannot suppress the deformation of the surrounding rock, increasing the length of the fully anchored bolt can improve the anchoring effect of the fully anchored bolt. However, increasing the length of the bolt cannot significantly improve the anchoring effect when the length of the fully anchored bolt exceeds a certain range.

\section{Conclusions}

Based on the deformation law of surrounding rock, the interactive model of the fully anchored bolt and the surrounding rock is established, and the analytical expressions of the axial force and shear stress of the fully anchored bolt during normal support process and failed support process are derived. The main conclusions can be summarized as follows:

(1) The stress distribution of the fully anchored bolt is consistent with the neutral point theory during the normal support process. The main factors affecting the stress distribution of the fully anchored bolt are the types of the surrounding rock, the anchoring parameters, and the support resistance, respectively.

(2) The influence of the surrounding rock properties on the stress distribution of the fully anchored bolt in the normal support process is mainly manifested as follows: the softer the surrounding rock is, the axial force and the shear stress at each position on the fully anchored bolt will increase accordingly. The main reason for this is the fact that the softer the surrounding rock is, the greater the deformation of the surrounding rock will be, and the deformation of the fully anchored bolt will increase, resulting in more opportunities for the fully anchored bolt to participate in suppressing the deformation of the surrounding rock of the roadway. Therefore, the important factor of surrounding rock properties should be considered when selecting the fully anchored bolt to support the roadway.

(3) The main influence of the support resistance on the stress distribution of the fully anchored bolt in the normal support process is mainly manifested in the increase of the support resistance, which will increase the axial force and shear stress of the fully anchored bolt and make the neutral point move toward the orifice on the bolt. Therefore, the support resistance is one of the main factors affecting the stress distribution of the fully anchored bolt; increasing the support resistance by changing the row spacing can improve the anchoring effect of the fully anchored bolt. However, when the fully anchored bolt support critically fails, although increasing the support resistance can reduce the stress distribution of the fully anchored bolt, the total reduction of stress decreases continuously. Therefore, when the existing supports cannot effectively control the deformation of the surrounding rock, the roadway cannot be reinforced only by increasing the support resistance and should be controlled in combination with other effective measures.

(4) The length of the bolt has a certain influence on the stress distribution of the fully anchored bolt. In the process of fully anchored bolt support, due to the timeliness of the interaction between the fully anchored bolt and the surrounding rock, increasing the length of the bolt can improve the support performance of the fully anchored bolt when it is within a reasonable range. However, increasing the length of the bolt beyond a certain range does not significantly improve the anchoring effect of the fully anchored bolt.

\section{Data Availability}

This paper contains all the data generated or published during the study; no other data were used to support this study.

\section{Conflicts of Interest}

The authors declare that they have no conflicts of interest.

\section{Acknowledgments}

This work was financially supported by the National Natural Science Foundation of China (Grants nos. 51874002, 51574006, 51774008, and 51774012).

\section{References}

[1] S.-H. Jiang, D.-Q. Li, L.-M. Zhang, and C.-B. Zhou, "Timedependent system reliability of anchored rock slopes considering rock bolt corrosion effect," Engineering Geology, vol. 175, no. 6 , pp. 1-8, 2014.

[2] E. Blanco-Fernandez, D. Castro-Fresno, J. J. Del Coz Diaz, and L. Lopez-Quijada, "Flexible systems anchored to the ground for slope stabilisation: critical review of existing design methods," Engineering Geology, vol. 122, no. 10, pp. 129-145, 2011.

[3] E. T. Brown, "Rock engineering design of post-tensioned anchors for dams-a review," Journal of Rock Mechanics and Geotechnical Engineering, vol. 7, no. 1, pp. 1-13, 2015.

[4] W. Yu, B. Pan, F. Zhang, S. Yao, and F. Liu, "Deformation characteristics and determination of optimum supporting time of alteration rock mass in deep mine," KSCE Journal of Civil Engineering, vol. 23, no. 11, pp. 4921-4932, 2019.

[5] J. S. Sim, T. S. Kang, Y. T. Lee, and H. J. Kim, "Analysis for mechanical behavior of GFRP rock bolt for permanent support of tunnel," Pharmacology \& Terapeutics, vol. 14, no. 6, pp. 91-105, 2010.

[6] W. Wang, Q. Song, C. Xu, and H. Gong, "Mechanical behaviour of fully grouted GFRP rock bolts under the joint action of pre-tension load and blast dynamic load," Tunnelling and Underground Space Technology, vol. 73, pp. 82-91, 2018.

[7] S. Ma, J. Nemcik, and N. Aziz, "Simulation of fully grouted rockbolts in underground roadways using FLAC2D," Canadian Geotechnical Journal, vol. 51, no. 8, pp. 911-920, 2014. 
[8] Q. Wang, B. Jiang, R. pan et al., "Failure mechanism of surrounding rock with high stress and confined concrete support system," International Journal of Rock Mechanics and Mining Sciences, vol. 102, no. 9, pp. 89-100, 2017.

[9] S.-C. Li, H.-T. Wang, Q. Wang et al., "Failure mechanism of bolting support and high-strength bolt-grouting technology for deep and soft surrounding rock with high stress," Journal of Central South University, vol. 23, no. 2, pp. 440-448, 2016.

[10] H. Kang, X. Zhang, S. Li, Y. Wu, and F. Gao, "In-situ stress measurements and stress distribution characteristics in underground coal mines in China," Engineering Geology, vol. 116, no. 3-4, pp. 333-345, 2010.

[11] H. P. Xie, F. Gao, Y. Ju et al., "Quantitative definition and Investigation of deep mining," Journal of China Coal Society, vol. 40, no. 1, pp. 1-10, 2015.

[12] C. C. Li, "A new energy-absorbing bolt for rock support in high stress rock masses," International Journal of Rock Mechanics and Mining Sciences, vol. 47, no. 3, pp. 396-404, 2010.

[13] C. C. Li, "Performance of D-bolts under static loading," Rock Mechanics and Rock Engineering, vol. 45, no. 2, pp. 183-192, 2012.

[14] W. Nie, Z. Y. Zhao, Y. J. Ning, and W. Guo, "Numerical studies on rockbolts mechanism using $2 \mathrm{D}$ discontinuous deformation analysis," Tunnelling and Underground Space Technology, vol. 41, pp. 223-233, 2014.

[15] D. Bernaud, S. Maghous, P. de Buhan, and E. Couto, “A numerical approach for design of bolt-supported tunnels regarded as homogenized structures," Tunnelling and Underground Space Technology, vol. 24, no. 5, pp. 533-546, 2009.

[16] F. Greco, L. Leonetti, and R. Luciano, "A multiscale model for the numerical simulation of the anchor bolt pull-out test in lightweight aggregate concrete," Construction and Building Materials, vol. 95, no. 10, pp. 860-874, 2015.

[17] A. Mortazavi and F. Tabatabaei Alavi, "A numerical study of the behavior of fully grouted rockbolts under dynamic loading," Soil Dynamics and Earthquake Engineering, vol. 54, pp. 66-72, 2013.

[18] S. Ding, H. Jing, K. Chen, G. A. Xu, and B. Meng, "Stress evolution and support mechanism of a bolt anchored in a rock mass with a weak interlayer," International Journal of Mining Science and Technology, vol. 27, no. 3, pp. 573-580, 2017.

[19] J. Liu, H. Yang, H. Wen, and X. Zhou, "Analytical model for the load transmission law of rock bolt subjected to open and sliding joint displacements," International Journal of Rock Mechanics and Mining Sciences, vol. 100, no. 1, pp. 1-9, 2017.

[20] Y. M. Zhao, N. Zhang, and X. G. Zhang, "Experimental study of axial stress distribution and transfer along the bolt rods in an underground coal mine," Arabian Journal of Geosciences, vol. 100, no. 1, pp. 1-9, 2017.

[21] X. Wu, Y. Jiang, G. Wang, B. Gong, Z. Guan, and T. Deng, "Performance of a new yielding rock bolt under pull and shear loading conditions," Rock Mechanics and Rock Engineering, vol. 52, no. 9, pp. 3401-3412, 2019.

[22] T. J. Freeman, "The behavior of fully-bonded rock bolts in the Kielder experimental tunnel," Tunnels and Tunnelling, vol. 10, no. 5, pp. 37-40, 1978.

[23] Y. L. Chen, J. Y. Teng, R. A. Sadiq, and K. Zhang, "Experimental study of bolt-anchoring mechanism for bedded rock mass," International Journal of Geomechanics, vol. 20, no. 4, Article ID 04020019, 2020.

[24] A. J. Hyett, M. Moosavi, and W. F. Bawden, "Load distribution along fully grouted bolts, with emphasis on cable bolt reinforcement," International Journal for Numerical and
Analytical Methods in Geomechanics, vol. 20, no. 7, pp. 517544, 2015.

[25] C. Yuan, L. Fan, J.-F. Cui, and W.-J. Wang, "Numerical simulation of the supporting effect of anchor rods on layered and nonlayered roof rocks," Advances in Civil Engineering, vol. 2020, Article ID 4841658, 14 pages, 2020.

[26] Y. Cai, T. Esaki, and Y. Jiang, "An analytical model to predict axial load in grouted rock bolt for soft rock tunnelling," Tunnelling and Underground Space Technology, vol. 19, no. 6, pp. 607-618, 2004.

[27] C. You, "Analysis of the force of full-length bond bolt," Chinese Journal of Rock Mechanics and Engineering, vol. 19, no. 3, pp. 339-341, 2000.

[28] C. Li and B. Stillborg, "Analytical models for rock bolts," International Journal of Rock Mechanics and Mining Sciences, vol. 36, no. 8, pp. 1013-1029, 1999.

[29] S. Q. Ma, Z. Y. Zhao, W. Nie, and X. Zhu, “An analytical model for fully grouted rockbolts with consideration of the pre- and post-yielding behavior," Rock Mechanics and Rock Engineering, vol. 50, no. 11, pp. 3019-3028, 2017.

[30] M. Blanco, M. Tijani, and F. Hadj-Hassen, "A new analytical solution to the mechanical behaviour of fully grouted rockbolts subjected to pull-out tests," Construction and Building Materials, vol. 25, no. 2, pp. 749-755, 2011.

[31] J. Chen, P. C. Hagan, and S. Saydam, "A new laboratory short encapsulation pull test for investigating load transfer behavior of fully grouted cable bolts," Geotechnical Testing Journal, vol. 41, no. 3, Article ID 20170139, 2018.

[32] P. Soparat and P. Nanakorn, "Analysis of anchor bolt pullout in concrete by the element-free Galerkin method," Engineering Structures, vol. 30, no. 12, pp. 3574-3586, 2008.

[33] Y. M. Li, C. X. Zhao, L. Cong, X. R. Meng, and C. L. Dong, "Analysis of stress distribution characteristics of fully anchored bolt based on actual surrounding rock deformation," Journal of China Coal Society, vol. 44, no. 10, pp. 2966-2973, 2019.

[34] S. L. Lu and Y. D. Jiang, "The control of support resistance over strata around roadway in soft rock," Rock and Soil Mechanics, vol. 19, no. 1, pp. 1-6, 1998.

[35] C. Yang, S. L. Lu, and Y. D. Jiang, "Control effect of support resistance on roadway deformation under different rock conditions," Journal of China University of Mining \& Technology, vol. 29, no. 2, pp. 170-173, 2000.

[36] S. L. Lu, G. B. Fu, and L. Tang, "Regularity of deformation of rocks around roadway under mining influence and change of rockbolt resistance," Journal of China University of Mining \& Technology, vol. 28, no. 3, pp. 201-203, 1999. 